\title{
In Vitro Activity of Cocoa Powder Extracts on Some Biomarkers Implicated in P. falciparum Malaria Pathogenesis
}

\author{
Seth Kwabena Amponsah ${ }^{1, *}$ and Nana Nim Dwumfour ${ }^{2}$ \\ ${ }^{1}$ Department of Pharmacology, University of Ghana Medical School, Korle-Bu, Ghana \\ ${ }^{2}$ Equinox Health Care, Ellicott City, Maryland, USA
}

\begin{abstract}
Malaria is a public health concern in many developing countries, including Ghana, and efforts are being made to eradicate it. Extracts from natural products have been used in several malaria endemic areas for malaria prophylaxis and treatment. Natural cocoa powder has been reported to possess in vitro direct inhibitory on $P$. falciparum. This study investigated the effect of natural cocoa powder on inducible nitric oxide, TNF- $\alpha$ and IL-10, biomarkers that play different roles in malaria pathogenesis. Chloroform and ethylacetate fractions of cocoa powder were cultured together with phytohemagglutinin-stimulated peripheral blood mononuclear cells (PBMCs) for 24 hours at $37^{\circ} \mathrm{C}$ with $5 \% \mathrm{CO}_{2}$. Cell free supernatants were harvested and assayed for iNO, TNF- $\alpha$ and IL-10. Inducible NO was determined by diazotization reaction developed by Griess. TNF- $\alpha$ and IL-10 were determined by ELISA. This study showed that TNF- $\alpha$ and iNO in phytohemagglutinin-stimulated PBMCs were significantly reduced by cocoa fractions $(p<0.05)$, but IL-10 levels did not change significantly $(p>0.05)$, although TNF- $\alpha$ is known to up-regulate IL-10. Apart from the documented direct inhibitory effect of cocoa powder on $P$. falciparum, it can be hypothesized that the antiplasmodial activity of unsweetened cocoa powder could also be due to its ability to significantly inhibit iNO and TNF- $\alpha$, inflammatory substances implicated in severe malaria pathogenesis.
\end{abstract}

Keywords: Antiplasmodial activity, Cytokines, fractions, Peripheral blood mononuclear cells, Supernatants.

\section{INTRODUCTION}

Malaria, caused by protozoan parasites of the genus Plasmodium, is a major parasitic disease in tropical and subtropical regions. It is estimated that 300-500 million people contract malaria resulting in $1.5-2.7$ million deaths annually [1, 2]. Thus, the control of malaria has become of much concern in many countries, especially in the third-world countries.

The past decade has witnessed an increased interest in the role of short-lived inducible nitric oxide (iNO) in P. falciparum malaria. Some researchers have associated iNO with severe and complicated malaria, particularly cerebral malaria [3, 4]. Cytokines and other macrophage secretory products are continually produced by human host in response to invading parasites. However, high concentrations often lead to deleterious effects on host tissues. Data also suggest that $\mathrm{IL}-10$, an anti-inflammatory cytokine, mediates immunostimulatory properties that help to eliminate infectious and noninfectious particles with limited inflammation [5]. A low IL-10/TNF ratio has been found to be associated with severe anemia during malaria episode [6].

Plant products have been reported to be good sources of antioxidants due to phenolic compounds

\footnotetext{
*Address correspondence to this author at the Department of Pharmacology, University of Ghana Medical School. P. O. Box 4236, Korle-Bu, Accra, Ghana;

Tel: +233-243-851461; E-mail: sethicom@yahoo.com
}

found in them [7]. Good sources of these phenols include tea, cocoa, wine, fruits and vegetables. However, cocoa has been found to be a rich source of these phenols [8]. Extracts of different cocoa byproducts have also shown strong antioxidant activity which is proportional to their flavonoid content [9]. Anecdotal reports indicate that a daily intake of a beverage of unsweetened cocoa powder protects an individual against $P$. falciparum malaria [10]. The direct inhibitory effect of natural cocoa powder on $P$. falciparum in vitro has been reported [11]. Aside this effect of cocoa on $P$. falciparum, other possible mechanisms responsible for its antiplasmodial activity needs to be investigated.

\section{MATERIALS AND METHODS}

\section{Preparation of Fractions}

One kilogram of Good food Natural Cocoa Powder (batch number KK 0901A; Kakawa Enterprise Ltd., Accra, Ghana) was put in a beaker and macerated with $98 \%(\mathrm{vol} / \mathrm{vol})$ methanol for 14 days. This was to aid in extracting flavonoids from the cocoa powder. The supernatant was filtered by suction, and the filtrate or crude methanol extract was evaporated to dryness under vacuum using a rotary evaporator (Rotavapor ${ }^{\circledR}$, Buchi, Flawil, Switzerland) at $40^{\circ} \mathrm{C}$. A yield of $12.8 \%$ was obtained. The crude methanol extract was dissolved in $200 \mathrm{~mL}$ of a water-methanol mixture (1:9). The aqueous-methanol solution formed was partitioned three times in succession with $100 \mathrm{~mL}$ each 
of petroleum ether, chloroform, ethyl acetate, and butanol. Each fraction was evaporated to dryness with the rotary evaporator.

A stock solution of $100 \mathrm{mg} / \mathrm{mL}$ was prepared for the crude methanol extract and the five cocoa fractions (aqueous-methanol, petroleum ether, chloroform, ethyl acetate, and butanol) with RPMI 1640 medium (SigmaAldrich Co. LLC, USA). To ensure sterility, each of the stock samples was filtered using a Millipore (Carrigtwohill, Ireland) filter (pore size, $0.22 \mu \mathrm{m}$ ).

\section{Human Peripheral Blood Mononuclear Cell (PBMC) Isolation and Culture with Cocoa Fractions}

Initial studies using similar extract and fractions showed that chloroform and ethyl acetate fractions of Natural Cocoa Powder had the greatest direct inhibitory activity on $P$. falciparum in vitro [11]. Based on bioassay-guided fractionation, these two fractions were cultured together with PBMCs.

After written informed consent was signed, whole blood was taken from 6 healthy volunteers into heparinized tubes. Ten milliliters of each blood sample was mixed with an equal volume of dilution medium $\{500 \mathrm{~mL}$ of RPMI $1640,5 \mathrm{~mL}$ of L-glutamine and $5 \mathrm{~mL}$ of Penicillin + Streptomycin $(10 \mathrm{mg} / \mathrm{mL})\}$. The diluted samples were transferred into pre-labeled Accuspin tubes pre-dosed with $15 \mathrm{~mL}$ of Ficoll-Paque solution. The tubes were centrifuged at $2000 \mathrm{rpm}$ for 10 minutes to obtain a middle ring of mononuclear cells above the separation disc. A serological pipette was used to pick up the mononuclear cells into centrifuge tubes containing $10 \mathrm{~mL}$ of PBMC wash medium $\{500 \mathrm{~mL}$ RPMI 1640, $5 \mathrm{~mL}$ L-glutamine, $5 \mathrm{~mL}$ Penicillin + Streptomycin $(10 \mathrm{mg} / \mathrm{mL})$ and $25 \mathrm{~mL}$ Fetal Bovine Serum\}. Each tube was centrifuged for 10 minutes at $2000 \mathrm{rpm}$ and supernatant discarded. The bottom of the tube was tapped to loosen the mononuclear cells. This process was repeated and the isolated PBMCs dispensed into tubes containing $5 \mathrm{~mL}$ re-suspension medium $\{500 \mathrm{~mL}$ RPMI 1640, $5 \mathrm{~mL}$-glutamine, $5 \mathrm{~mL}$ Penicillin + Streptomycin $(10 \mathrm{mg} / \mathrm{mL}), 50 \mathrm{~mL}$ Fetal Bovine Serum\}. Twenty microliters of this was dispensed into eppendorf tubes containing $180 \mu \mathrm{L}$ of $0.4 \%$ trypan blue. A hemacytometer was used to estimate cell viability and total number of PBMCs. It was ensured that the isolated cells had a percent viability greater than $96 \%$ and a cell count of $2 \times 10^{6}$ cells $/ \mathrm{mL}$. Five hundred microliters of isolated PBMC was dispensed into 24-well plates.

Five hundred microliters of re-suspension media was added to an equal volume of the PBMCs, and this served as the control (first treatment). Two hundred microliters of phytohemagglutinin (PHA) of concentration $5 \mu \mathrm{g} / \mathrm{mL}$ and $300 \mu \mathrm{L}$ of re-suspension media were added to another set of isolated PBMC (second treatment). The third treatment had $200 \mu \mathrm{L}$ of PHA $(5 \mu \mathrm{g} / \mathrm{mL})$ and $300 \mu \mathrm{L}(100 \mathrm{mg} / \mathrm{mL})$ of the chloroform (CF) of cocoa powder. The fourth had 200 $\mu \mathrm{L}$ of PHA $(5 \mu \mathrm{g} / \mathrm{mL})$ and $300 \mu \mathrm{L}(100 \mathrm{mg} / \mathrm{mL})$ of the ethyl acetate fractions (EAF) of cocoa powder. After incubation of PBMC with PHA and cocoa fractions, cell viability was measured again by the trypan blue exclusion method. No toxicity could be observed at the concentration range applied. Cell-free supernatants were harvested after 24 hours of incubation $\left(37^{\circ} \mathrm{C}\right.$ with $5 \% \mathrm{CO}_{2}$ ) and stored at $-80^{\circ} \mathrm{C}$ until assayed for iNO, TNF- $\alpha$ and IL-10.

\section{Determination of Inducible Nitric Oxide in Culture Supernatant}

Inducible NO was assayed as nitrite, a stable metabolite of NO. The assay relies on a diazotization reaction developed by Griess [12]. The nitrite standard with an initial concentration of $0.1 \mathrm{M}$ was diluted 1000 times with re-suspension media to give a final concentration of $100 \mu \mathrm{M}$. Two-fold serial dilutions of the prepared standard were made to get a range of concentrations from 100 to $1.56 \mu \mathrm{M}$. Fifty microliters of each standard sample (supernatant) was dispensed in duplicates into a 96-well microtiter plate. The same volume of culture supernatant $(50 \mu \mathrm{L})$ of cell free PBMC collected after 24 hours was also dispensed in duplicates into the remaining wells of the plate. An equal volume of the Griess reagent (Sigma, USA) was dispensed into each well and incubated at room temperature for 15 minutes, protected from light. The optical densities were read immediately at $540 \mathrm{~nm}$ with a plate reader (Biotek Technologies, USA).

\section{Analysis for TNF- $\alpha$ and IL-10 in Culture Supernatant}

TNF- $\alpha$ and IL-10 in culture supernatant were measured using ELISA (Bender Medsystems, Austria) according to manufacturers' protocol. Optical densities were read immediately at $450 \mathrm{~nm}$ with the plate reader (Biotek Technologies, USA). All supernatants collected were run undiluted.

\section{Data Analysis}

All results were expressed as mean \pm standard error of the mean (SEM). Results were further compared by one-way analysis of variance (ANOVA) 
followed by Post Hoc analysis (Bonferroni) using Microsoft Statistical Package for the Social Sciences (SPSS) version 16.0. Statistical significance was taken as $p<0.05$.

\section{Ethical Issues}

This work was approved by the Ethical and Protocol Review Committee of the University of Ghana Medical School.

\section{RESULTS}

\section{Inducible NO in Culture Supernatant of Human PBMCs}

Mean nitrite concentration in PBMC only (control) assayed after 24 hours of incubation $(n=6)$ was $0.7 \pm$ 0.06 (SEM) $\mu \mathrm{M}$ and this increased to $10.6 \pm 1.26$ (SEM) $\mu \mathrm{M}$ when induced with PHA. The mean nitrite concentration decreased when incubated with chloroform and ethyl acetate fractions of unsweetened cocoa powder to $3.6 \pm 0.14$ (SEM) $\mu \mathrm{M}$ and $2.9 \pm 0.29$ (SEM) $\mu \mathrm{M}$ respectively (Figure 1). A significant difference $(p<0.05)$ was observed between the treatments after analysis with one-way ANOVA. The decrease in nitrite concentration observed for the two cocoa samples was found to be significant after Bonferroni's post hoc analysis $(p<0.001)$. However, the difference between mean nitrite concentrations in supernatant that had chloroform and ethyl acetate fractions of cocoa was not significant $(p=0.136)$.

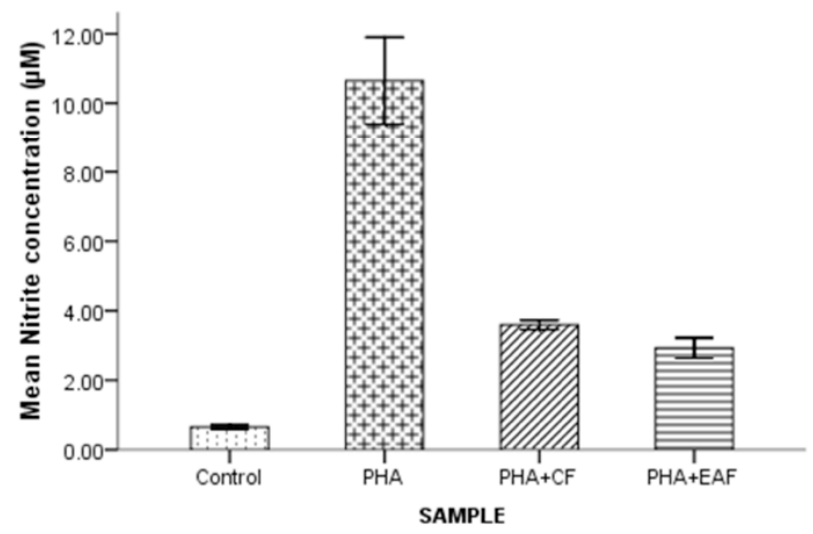

Figure 1: Nitrite concentration in PBMC after 24 hours of incubation.

\section{TNF- $\alpha$ in Culture Supernatant of Human PBMCs}

A mean TNF- $\alpha$ concentration of $223.6 \pm 7.7$ (SEM) $\mathrm{pg} / \mathrm{mL}$ was recorded for non-induced PBMC (control) after 24 hours of incubation $(n=6)$. When PBMC was stimulated with $\mathrm{PHA}$, the concentration increased to $564.9 \pm 8.9$ (SEM) pg/mL. TNF- $\alpha$ in stimulated PBMCs decreased when cultured together with cocoa fraction as shown in Figure 2. The chloroform fraction had the lower of the two fractions, with a TNF- $\alpha$ concentration of $435.4 \pm 8.9$ (SEM) $\mathrm{pg} / \mathrm{mL}$. One-way ANOVA and post hoc analysis revealed that the decrease recorded when stimulated PBMC was cultured with cocoa fractions was significant $(p<0.05)$.

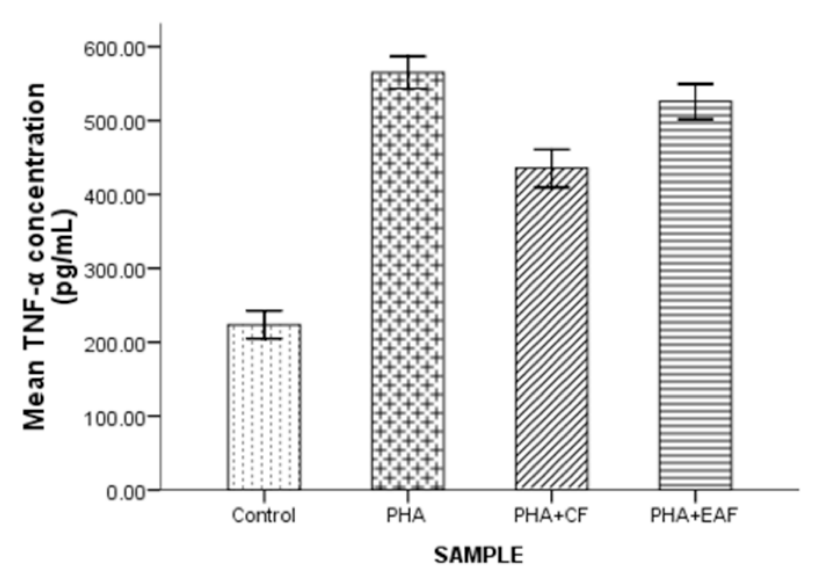

Figure 2: TNF-a in PBMC after 24 hours of incubation.

\section{IL-10 in Culture Supernatant of Human PBMCs}

Using one-way ANOVA, it was discovered that there was a significant difference $(p<0.05)$ in the mean IL10 concentration between PBMC treatments after 24 hours of incubation. Post hoc analysis however showed that the increase in IL-10 concentration after addition of ethyl acetate fraction of cocoa to induced PBMCs was not significant $(p>0.05)$, as shown in Figure 3 . The decrease observed after addition of chloroform fraction of cocoa in PHA-induced PBMC supernatant after 24 hours was also not significant $(p>0.05)$.

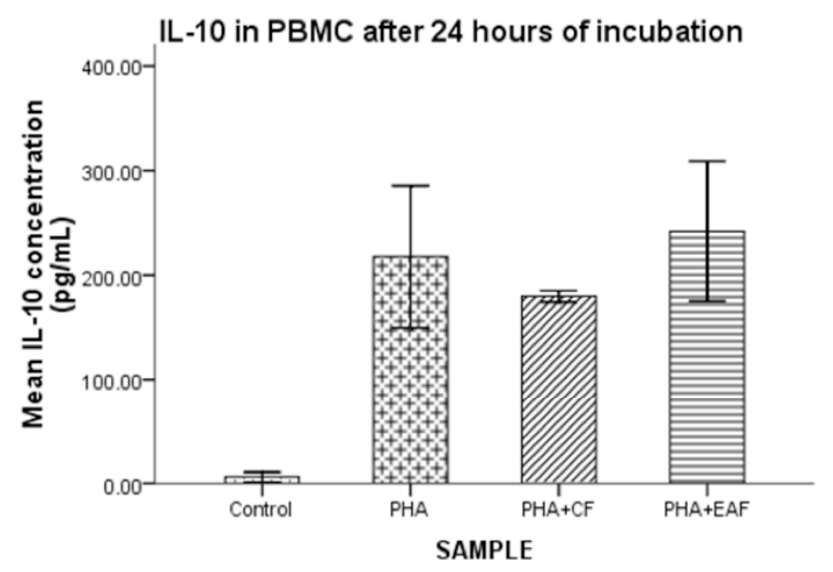

Figure 3: IL-10 in PBMC after 24 hours incubation.

\section{DISCUSSION}

Inducible nitric oxide synthase (iNOS) is an enzyme responsible for the production of inducible NO (iNO). 
This enzyme can produce micromolar concentrations of iNO, and such concentrations show cytotoxicity and tissue damage [13]. High concentrations of iNO and its derivatives, such as peroxynitrite and nitrogen dioxide, play important roles in inflammation and in multistage processes of carcinogenesis [14]. Chloroform and ethyl acetate fractions of cocoa powder significantly decreased iNO in the form of nitrite in human PBMC after stimulation with phytohemagglutinin (PHA). Some researchers have proposed that iNO contributes to the development of severe and complicated malaria [3]. Keller et al. showed that ex vivo and in vitro NO synthase activity in PBMCs is significantly elevated in children with malaria [15]. If indeed high levels of iNO have a role to play in severe malaria, then the ability of cocoa to reduce its levels as demonstrated in this study, could prove beneficial in preventing severe forms of $P$. falciparum malaria.

TNF- $\alpha$ is one of the major pro-inflammatory cytokines involved in the pathogenesis of chronic inflammatory diseases and is modulated by oxidative stress $[16,17]$. It is a key endogenous pyrogen in fever responses as has been verified in malarial infections $[18,19]$. In the present study, TNF- $\alpha$ increased significantly in human PBMC culture supernatant when induced with PHA. The addition of chloroform and ethyl acetate fractions to the induced PBMC cultures showed a significant decrease in TNF- $\alpha$ concentration. It has also been shown that the production of TNF- $\alpha$ is crucial for the synergistic induction of NO synthesis in stimulated macrophages [20]. The effect of the chloroform and ethyl acetate fractions of unsweetened cocoa powder on these two inflammatory molecules further supports the claim that plants containing flavonoids inhibit both iNO and TNF- $\alpha$ production. Furthermore, Kwiatkowski et al. found out that administering anti-TNF therapy to individuals with cerebral malaria reduced fever symptoms [21].

In the current study, culture supernatant of isolated PBMC stimulated with PHA showed a significant increase in IL-10 concentration compared with unstimulated (control). However, addition of cocoa fractions to the stimulated PBMC showed a decrease with the chloroform fraction but an increase with the ethyl acetate fraction. These changes were not statistically significant. It is worth noting that IL-10 is important in the down-regulation of inflammatory responses, and it has been shown that a low plasma concentration of IL-10 correlates with the occurrence of cerebral malaria and anemia in $P$. falciparum infections [6].

\section{CONCLUSION}

In summary, apart from its direct in vitro inhibitory effect on $P$. falciparum growth, the antiplasmodial activity of unsweetened cocoa powder can be postulated to be partly due to its ability to significantly inhibit iNO and TNF- $\alpha$, inflammatory substances implicated in the pathophysiology of severe malaria. However, unsweetened cocoa powder did not show a significant effect on the levels of the anti-inflammatory cytokine IL-10 in this study. Further studies to isolate, purify and identify active compounds responsible for these properties observed in this research need to be undertaken.

\section{ACKNOWLEDGEMENTS}

The authors are grateful to the staff of the Department of Pharmacology, University of Ghana Medical School, for technical, material, and financial support. Thanks also go to the College of Health Sciences, University of Ghana, for financial and institutional support.

\section{REFERENCES}

[1] Muentener P, Schlagenhauf P, Steffen R. Imported malaria (1985-95): trends and perspectives. Bull World Health Organ 1999; 77: 560-6. http://dx.doi.org/10.1038/415680a

[2] Sachs J, Malaney P. The economic and social burden of malaria. Nature 2002; 415: 680-5.

[3] Agbenyega T, Angus B, Bedu-Addo G, et al. Plasma nitrogen oxides and blood lactate concentrations in Ghanaian children with malaria. Trans R Soc Trop Med Hyg 1997; 91: 298-302. http://dx.doi.org/10.1016/S0035-9203(97)90083-3

[4] Burgner D, Xu W, Rockett $\mathrm{K}$, et al. Inducible nitric oxide synthase polymorphism and fatal cerebral malaria. Lancet 1998; 352: 1193-4. http://dx.doi.org/10.1016/S0140-6736(05)60531-4

[5] Asadullah K, Sterry W, Volk HD. Interleukin-10 therapy Review of a New Approach. Pharmacol Rev 2003; 55: 241269.

http://dx.doi.org/10.1124/pr.55.2.4

[6] Othoro C, Lal AA, Nahlen B, Koech D, Orago ASS, Udhayakumar V. A low interleukin-10 tumor necrosis factor$\alpha$ ratio is associated with malaria anemia in children residing in a holoendemic malaria region in western Kenya. J Infect Dis 1999; 179: 279-282.

http://dx.doi.org/10.1086/314548

[7] Guardia T, Rotelli EA, Juarez AO, Pelzer LE. Antiinflammatory properties of plant flavonoids. Effects of rutin, quercetin and hesperidin on adjuvant arthritis in rat. IL Farmaco 2001; 56: 683-7.

http://dx.doi.org/10.1016/S0014-827X(01)01111-9

[8] Hammerstone JF, Lazarus SA, Schmitz HH. Procyanidin content and variation in some commonly consumed foods. $J$ Nutr 2000; 130: 2086-2092.

[9] Azizah AH, Ruslawati Nik MN, Tee Swee T. Extraction and characterisation of antioxidants from cocoa by-product. Food Chem 1999; 64: 199-202.

http://dx.doi.org/10.1016/S0308-8146(98)00121-6 
[10] Addai FK. Natural cocoa as diet-mediated antimalarial prophylaxis. Med Hypotheses 2010; 74: 825-830. http://dx.doi.org/10.1016/j.mehy.2009.12.003

[11] Amponsah SK, Bugyei KA, Osei-Safo D, et al. In vitro activity of extract and fractions of natural cocoa powder on Plasmodium falciparum. J Med Food 2012; 15: 476-482. http://dx.doi.org/10.1089/jmf.2011.0220

[12] Griess JP. Determination of nitrite and nitrate by Griess reaction. Ber Deutsch Chem Ges 1897; 12: 426. http://dx.doi.org/10.1002/cber.187901201117

[13] Ohshima $\mathrm{H}$, Bartsch $\mathrm{H}$. Chronic infections and inflammatory processes as cancer risk factors: possible role of nitric oxide in carcinogenesis. Mutat Res 1994; 305: 253-264. http://dx.doi.org/10.1016/0027-5107(94)90245-3

[14] Halliwell B. Free radicals, antioxidants, and human disease: curiosity, cause, or consequence? Lancet 1994; 344: 721-5. http://dx.doi.org/10.1016/S0140-6736(94)92211-X

[15] Keller CG, Kremsner PG, Hittner JB, Misukonis AM, Weinberg BJ, Perkins DJ. Elevated nitric oxide production in children with malarial anemia: Hemozoin-induced nitric oxide synthase Type 2 transcripts and nitric oxide in blood mononuclear cells. Infect Immun 2004; 72: 4868-4873. http://dx.doi.org/10.1128/IAl.72.8.4868-4873.2004

[16] Calamia KT. Current and future use of anti-TNF agents in the treatment of autoimmune, inflammatory disorders. Adv Exp Med Biol 2003; 528: 545-9.

http://dx.doi.org/10.1007/0-306-48382-3 110
[17] Taylor PC, Williams RO, Feldmann M. Tumour necrosis factor alpha as a therapeutic target for immune-mediated inflammatory diseases. Curr Opin Biotechnol 2004; 15: 557 563.

http://dx.doi.org/10.1016/j.copbio.2004.09.005

[18] Karunaweera ND, Grau GE., Gamage P, Carter R, Mendis $\mathrm{KN}$. Dynamics of fever and serum TNF levels are closely associated during clinical paroxysms in Plasmodium vivax malaria. Proc Natl Acad Sci 1992; 89: 3200-3.

http://dx.doi.org/10.1073/pnas.89.8.3200

[19] Mendis KN, Carter R. Clinical disease and pathogenesis in malaria. Parasitol Today 1995; 11: 1-16. http://dx.doi.org/10.1016/0169-4758(95)80143-X

[20] Park YC, Rimbach G, Saliou C, Valacchi G, Packer L. Activity of monomeric, dimeric, and trimeric flavonoids on NO production, TNF- $\alpha$ secretion, and NF-KB-dependent gene expression in RAW 264.7 macrophages. FEBS Lett 2000; 465: 93-7.

http://dx.doi.org/10.1016/S0014-5793(99)01735-4

[21] Kwiatkowski D, Molyneux ME, Stephens S, Curtis N, Klein N, Pointaire P, Smit M, Allan R, Brewster DR, Grau GE, Greenwood BM. Anti-TNF therapy inhibits fever in cerebral malaria. Q J Med 1993; 86: 91-8. 